\title{
A Framework of Privacy Preserving QOS Prediction used for Web Service Recommendation
}

\author{
Bhakti Chudiwal $^{1}$, P. Jawalkar ${ }^{2}$ \\ ${ }^{1}$ Pune University, JSPM (BSIOTR), Wagholi, 411005 \\ ${ }^{2}$ Professor, Pune University, JSPM (BSIOTR), Wagholi, 411005
}

\begin{abstract}
With increasing existence and reception of Web services on the World Wide Web, Quality-of-Service (QoS) is becoming essential for unfolding nonfunctional characteristics of Web services. Collaborative filtering (CF) techniques are becoming progressively popular with the progress of the Internet. To demeanor collaborative filtering, data from customers are needed. Though, gathering high quality data from customers is a difficult task because many customers are so concerned about their privacy that they might choose to give fabricated information. We propose a randomized disconcertion technique to safeguard user's privacy while still constructing accurate recommendations. Then, based on the collected QoS data, a collaborative filtering approach is designed to calculate Web service QoS.
\end{abstract}

Keywords: QoS prediction; collaborative filtering; privacy preservation; Web service recommendation.

\section{Introduction}

Web service is mode of communication between two electronic devices over network. In business and large scale enterprises the applications of web services are being used. Nowadays, in businesses these applications have elevated from huge application to dynamic setup of business processes. In recent scenario, web services are broadly used in industry and academia. It is a collection of open protocols and used for substituting data between applications or systems. Software applications are inscribed in different types of programming languages and running on diverse platforms which can be used in web services to exchange data over computer networks like the Internet. Web Services have substantial characteristics like they are platform independent, modular, dynamic applications, self-contained, highly interoperable, portable, language independent, and having well defined services.

In SOA (service- oriented application), users request the service from the server. Servers get the complete request from users. Previously the server gets the complete request from users this request goes through the service brokers. When implementing SOA, service users usually get a list of web services from service brokers or search engines that will encounter the specific functional requirements. It needs to recognize the ideal one from the functionally equivalent candidates. It is tough to select the best performing one. It has distributed to service selection and recommendation which is instantly needed.

Quality of service is to signify the non-functional performance of web service and it has dispensed service selection. QoS is defined as a set of user aware properties including reputation, availability and response time etc. It is difficult for users to obtain QoS information by assessing all the service candidates, since it is conducting real-world web service requests which takes extensive time and is resourceconsuming. QoS plays a vital role in Web service recommendation, conferring to which analogous services can be ranked and carefully chosen for users. Service invocations usually depend on on the Internet for connectivity and are profoundly influenced by the dynamic network conditions.

In recent years, with the advance of Internet technology, the QoS of service-oriented systems has become a significant research topic to shape high quality systems, and it has captivated extreme amounts of consideration from both academia and industry.

\section{Literature Survey}

\subsection{Web Service Recommendation based QOS}

QoS-based Web service recommendation has lately engrossed much consideration from the service community, for providing a encouraging way to help users select highquality services out of all the candidate services conferring to the user imaginary QoS values. The more QoS information the user contributes, the more precise service recommendations the user can obtain, since more user characteristics can be scrutinized from the user added information. In web service recommender system, users usually offer QoS values on a insignificant number of web services. Traditional memory-based CF algorithms lament from the infrequent user added data set, since it's tough to find analogous users without adequate knowledge of their service experience.

The elementary function of this architecture is to obtain appropriate Web service QoS information from different service users critical for making precise Web service recommendations. The idea is that by funding the independently observe Web service QoS information to Web Service Recommender System, the service users can gain precise Web service recommendation service 
International Journal of Science and Research (IJSR)

ISSN (Online): 2319-7064

Index Copernicus Value (2013): 6.14 $\mid$ Impact Factor (2014): 5.611

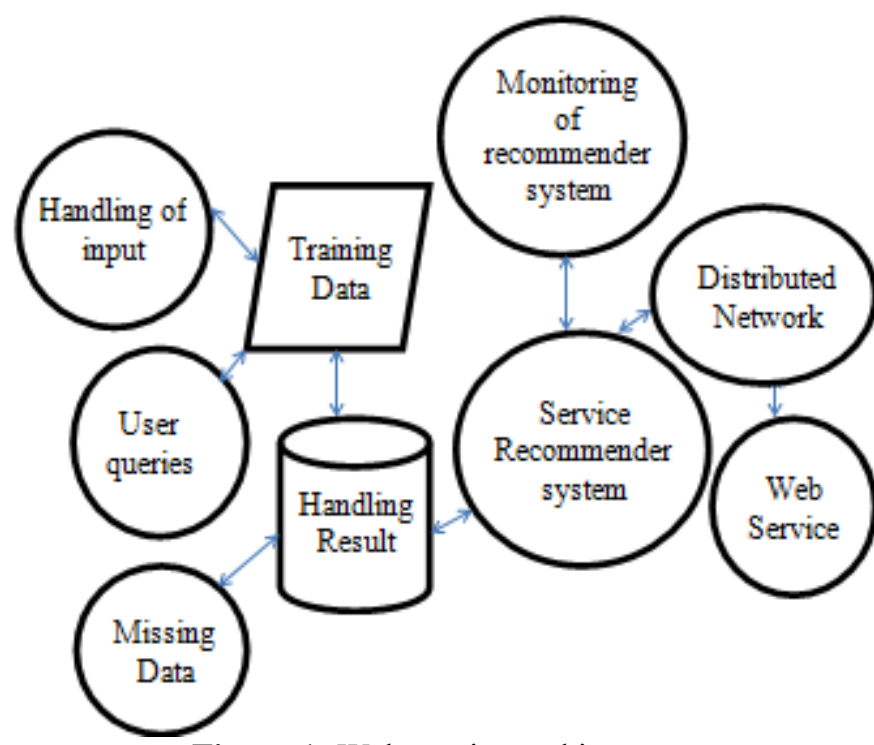

Figure 1: Web service architecture

\subsection{Filter approach used for QOS prediction}

\subsubsection{Collaborative Filtering}

Web service QoS value calculation is formed by novel hybrid cooperative filtering algorithm[5]. They have established that WSRec[1] get the well expectancy accurateness as compare to other methods. There are some service user-perspective has the following difficulties:

1) It needs service calls; it performed the overheads of the service users. Also, it consumes possessions of the service providers.

2) It may evaluation too many service applicants. It may not expose some proper Web services to the service users.

The estimate of web service is not dedicated for service user. It used method which is combination Cooperative filtering method with the help of this method they can decrease the above difficulties. It practices the new combination cooperative filtering algorithm for Web service. The recommendation value correlating with other out dated supportive filtering methods.

As we know, CF (Collaborative Filtering), also known as social filtering or social information filtering, is the most popular technique in the field of personalized recommender systems. It aims to predict and identify the information (e.g., website, commodity, social networking service, etc.) a user might be interested in according to historical data, and to make recommendations on this basis. To the best of our knowledge, there are mainly two types of classic CF methods for Web service recommendation: memory-based and modelbased approaches.

\subsubsection{Matrix factorization model}

It is usually working in collaborative filtering techniques crops dense user and dense item factor matrices the supposition is that users have a likeness towards all hidden factors and items possess all hidden factors. Matrix factorization (MF)[3] is one of the most recognized model based CF approaches, which is to activity the dormant factors that can determine QoS equally from the user and the service aspects. As a typical model-based approach in collaborative filtering, matrix factorization models are accurate and scalable in many applications. When employing
MF to make QoS predictions[4], a low-rank valuation to the unique target medium is desired.

\subsubsection{Privacy Issues}

\section{Privacy in Service Computing}

In service computing, applications are normally made by binding web services obtainable by different service providers. User information repeatedly needs to be shared across the providers to satisfy a complete application task. This can surge privacy issues between users and service providers when the selected Web services for structure have privacy procedures that are not amenable with users' privacy requirements.

\section{Privacy in Recommender Systems}

In recommender systems[6], users want to improve useful endorsements without assigning their privacy. To accomplish so, a variety of privacy-preserving obliging filtering methods have been suggested by using techniques such as randomization, cryptography and so on. Privacy is also of energetic importance to the appreciation of QoS-based Web service recommendation.

\subsubsection{Privacy Preserving Techniques}

\section{Heuristic-based techniques:}

An adaptive modification that adjusts only selected values that lessen the effectiveness loss rather than all available values.

\section{Cryptography-based techniques:}

This technique comprises secure multiparty computation where a computation is protected if at the accomplishment of the computation, no one can know everything except its own input and the results. Cryptography-based algorithms are deliberated for protective privacy in a dispersed situation by using encryption techniques.

\section{Reconstruction-based techniques:}

Here, the original dispersal of the data is reconvened from the randomized data.

\section{System Design}

\subsection{Structure of Privacy Preserving Web Service Recommendation:}


International Journal of Science and Research (IJSR)

ISSN (Online): 2319-7064

Index Copernicus Value (2013): 6.14 | Impact Factor (2014): 5.611

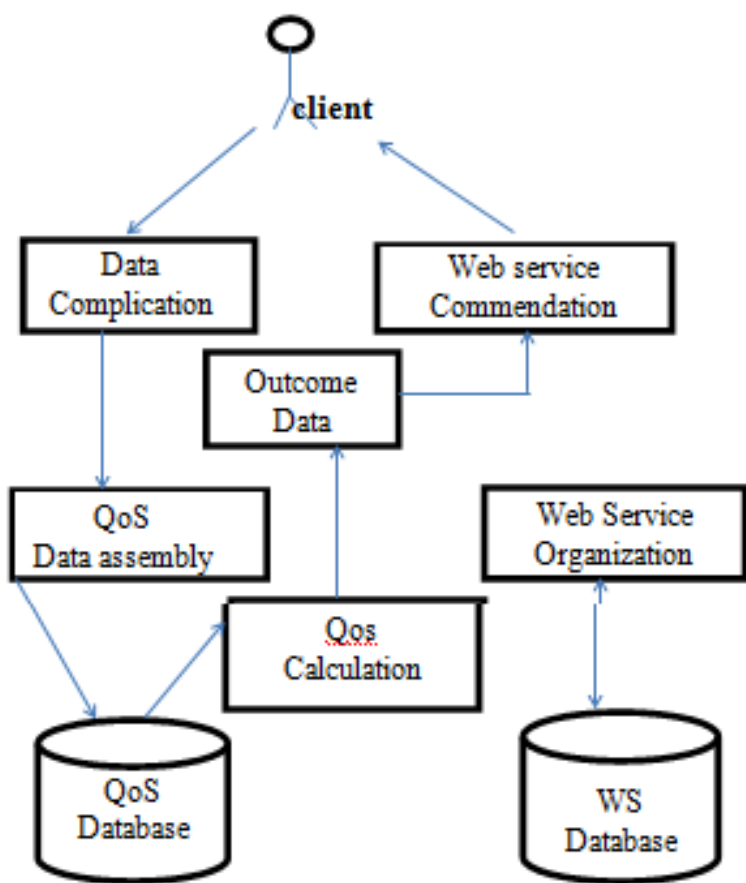

Figure 2: Structure of Privacy Preserving Web Service Recommendation

Framework permits users with superior control on their secretive usage data and less requirement on the server for privacy preservation. User privacy is conserved by our framework because:

a) For each user, user data are complicated before being acquiesced to the server, and the complication settings are only known to the user itself;

b) For the server, concerted QoS prediction is performed based exclusively on the complicated user data, whereby user-observed real QoS values cannot be inferred.

Commendation systems transformed the way inert websites communicate with their users. Rather than providing a static experience in which users search for theoretically buy products, recommender systems increase collaboration to deliver a more affluent experience. Recommender systems classify recommendations autonomously for individual users centered on past purchases and searches, and on other users' behavior. This article familiarizes you to recommender systems and the algorithms that they implement. The basic function of this architecture is to find adequate Web service QoS information from diverse service users crucial for making exact Web service recommendations. The idea is that by donating the individually observe Web service QoS information to Web Service Recommender System, the service users can obtain precise Web service reference service.

\section{System Algorithm}

It is challenging to mine the individualities of Web service QoS values, and the prophecy precision of previous algorithms cannot be reliable without believable and sufficient real-world Web service QoS data.

- The computational complexity of predicting one unknown QoS value using prediction algorithm.

- These outcomes are the theoretical foundation of our anticipated algorithm HAPA. HAPA includes user based and item based estimate rendering to results.

- All comparisons are calculated in development since it is very timewasting with a large dataset. Therefore resemblances calculations are not encompassed in the convolution of our prediction algorithm.

- To authorize the accurateness of our algorithm, we foresee only the known values, so that we can estimate the error between the prophesied values and real values.

A central issue is to regulate which ones midst these privacypreserving techniques better protect delicate information. However, this is not the solitary criteria with respect to which these algorithms can be evaluated. It is also significant to measure the quality of the data resultant from the variations pragmatic by each algorithm, as well as the presentation of the algorithms.

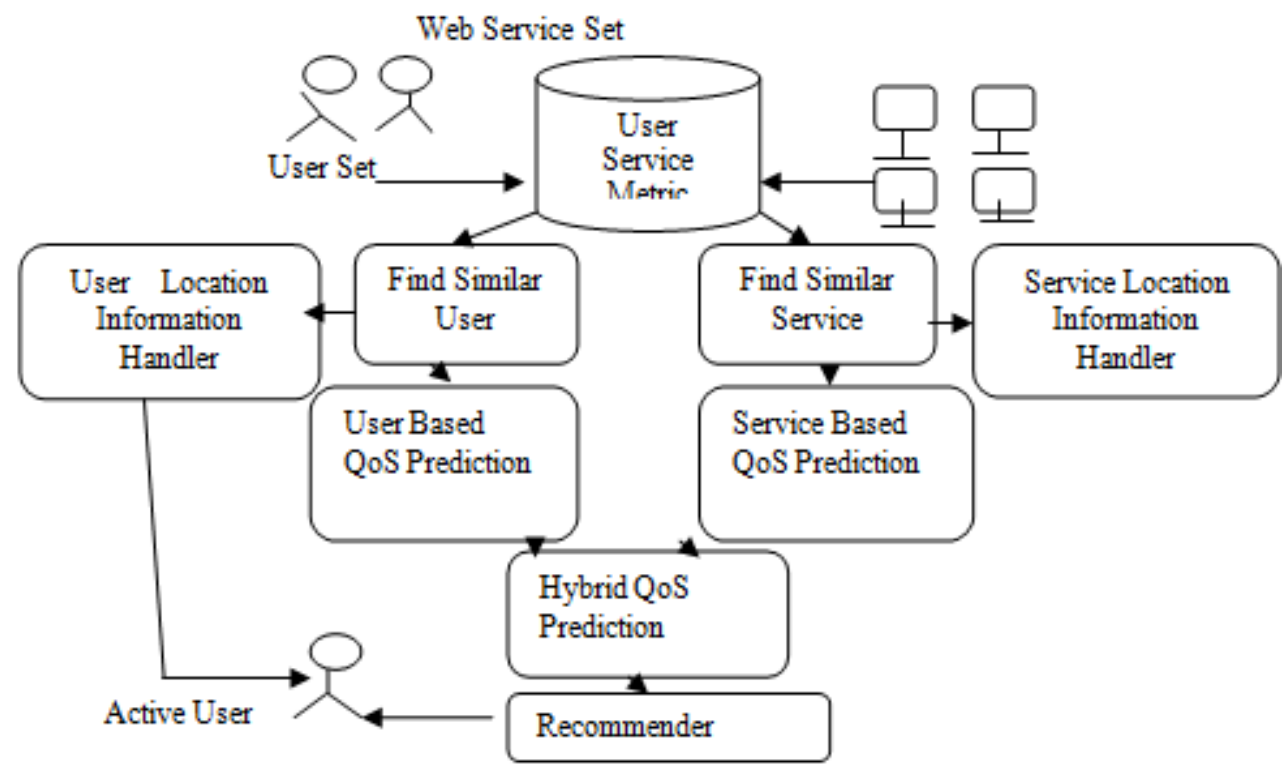

Figure 3: System Architecture 


\section{International Journal of Science and Research (IJSR) ISSN (Online): 2319-7064}

Index Copernicus Value (2013): 6.14 | Impact Factor (2014): 5.611

\section{Methods Used in QoS Prediction Approach}

\section{A. Data Complication}

The necessity for privacy preservation has directed to the advance of a number of data complication techniques, such as data encryption, data randomization. Due to the minor amount of data I make use of data randomization, a simple yet effective way to complicate the data.

\section{B. Z-Score Normalization:}

I perform Z-score normalization on the pragmatic QoS data as the first step. Z-score normalization is a standard normalization method to alter the data average and data variance.

\section{Data Randomization}

I accomplished randomized discomposure on the normalized QoS vector.

\section{Privacy-Preserving PMF (P-PMF)}

PMF, or probabilistic matrix factorization[1][3], as a standard model-based collaborative filtering approach. PMF mechanisms is a critical assumption of the low-rank structure of the QoS matrix. A matrix has a low rank when the admittances of the matrix are mostly correlated.

We propose to develop a framework that practices existing information (ratings and/or metadata) to produce high quality recommendations. Our model comprises classifying users and items into numerous overlapping clusters, based on label information derived from existing metadata. For example, users may be characterised established on their demographic profile; similarly, items can be characterised in agreement with the group they belong to. The class cataloguing is not restraining and a user/item can rapidly belong to multiple classes. Our main model recovers the concealed factor matrices for users and items such that in addition to sustaining the data reliability restrictions they are also reliable with the class label information.

By familiarizing administered learning into the BCS based matrix factorization model, we are able to progress retrieval correctness by effect of dropping data sparsely. Our model works well even for partial cold start condition. In such cases, the inadequate rating information is appropriately supported by use of available metadata to create more correct calculations as compared to using rating information alone.

A modest postponement of our principal construction presents an explanation to the pure (item and user) cold start problem as well. We learn a label consistency map concerning user or item label data to their concealed factor vectors - virtually on the side lines, while resolving our main formulation. This label steadiness map along with supporting data of new users/items can be used to make expectations for them. Our suggestion is based on the confidence that user allocation similar arrangement profiles will ascent to protest similar preferences. Similarly, items fit in to the shared type will possess similar covert factor vectors.

Thus, label consistency map learned for surviving users or items is equally appropriate to new users and items as well. Present works objective the two problems - correctness enhancement for warm start users and rating forecast for cold start scenario independently. There is no current model, to the best of our knowledge, which challenges to solve both the related problems in a mutual framework. Also, most of prevailing literature solves incomplete cold start problem (using some amount of rating data with auxiliary information), whereas we need only auxiliary data for new users or items. Thus, our design permits high estimate correctness for remaining as well as new users within the similar efficient framework. We also descend an wellorganized algorithm for our recommended formulation.

\section{Conclusion}

The assembly of the different QoS properties is significant for the accomplishment of the web service technology. Due to the growing popularity of Web services technology and the latent of dynamic service selection and integration, multiple service providers are now providing parallel services. QoS is a changed factor to discriminate functionally similar Web services. To make rather more problematic to comprehend is the progression of hiding unique data with arbitrary characters or data. Web service commendation helps users to find a mandatory service has become significant subject in service computation. The recommender system is proficient of cautionary or providing feedback to the end-users and supplementary make better conclusions about their privacy options. I make an determination with privacy preserving web service recommendation problem. I hope that this work can give a appropriate explanation to the user.

\section{Future Scope}

In our future work, more real-world Web services will be tested and more QoS properties of Web services will be measured. The procedure of the anticipated QoS values and the permutation of different QoS properties will be used. Intensification the basic Knowledge Bases to cover more measures related to privacy.Area Explicit Knowledge Bases to comprise more sub-domains and their associations. Discover application of other data attributes that are accessible by users such as: user response about quality of historical recommendations, more broad historic data, and user online activities.

- Method user studies and understands how careful users find the recommendations.

- Instrument the recommender system for dissimilar platforms (e.g. mobile) and make it existing to other researchers and consultants.

\section{References}

[1] Z. Zheng, H. Ma, M. R. Lyu, and I. King, "WSRec: A collaborative filtering based web service recommender system," in Proc. of the IEEE International Conference on Web Services (ICWS), 2009, pp. 437-444

[2] X. Su and T. M. Khoshgoftaar, "A survey of collaborative filtering techniques," Adv. Artificial Intelligence, vol. 2009, 2009.

[3] V. Nikolaenko, S. Ioannidis, U. Weinsberg, M. Joye, N. Taft, and D. Boneh, "Privacy-preserving matrix

\section{Volume 4 Issue 12, December 2015}




\section{International Journal of Science and Research (IJSR) \\ ISSN (Online): 2319-7064}

Index Copernicus Value (2013): 6.14 | Impact Factor (2014): 5.611

factorization," in Proc. of the ACM SIGSAC Conference on Computer \& Communications Security (CCS), 2013, pp. 801-812

[4] L. Shao, J. Zhang, Y. Wei, J. Zhao, B. Xie, and H. Mei, "Personalized QoS prediction for web services via collaborative filtering," in Proc. the IEEE International Conference on Web Services (ICWS), 2007, pp. 439446

[5] J. Canny, "Collaborative filtering with privacy," in Proc. of the IEEE Symposium on Security and Privacy, 2002, pp. $45-57$.

[6] J. Zhan, C. Hsieh, I. Wang, T. Hsu, C. Liau, and D. Wang, "Privacy preserving collaborative recommender systems," IEEE Transactions on Systems, Man, and Cybernetics, Part C, vol. 40, no. 4, pp. 472-476, 2010.

[7] E. Costante, F. Paci, and N. Zannone, "Privacy-aware web service composition and ranking," in Proc. of the IEEE International Conference on Web Services (ICWS), 2013, pp. 131-138.

[8] S. Tbahriti, C. Ghedira, B. Medjahed, and M. Mrissa, "Privacy enhanced web service composition," IEEE T. Services Computing, vol. 7, no. 2, pp. 210-222, 2014

[9] W. Lo, J. Yin, S. Deng, Y. Li, and Z. Wu, "An extended matrix factorization approach for QoS prediction in service selection," in Proc. of the 9th IEEE International Conference on Services Computing (SCC), 2012, pp. 162-169

[10]A. Bilge, C. Kaleli, I. Yakut, I. Gunes, and H. Polat, “A survey of privacy-preserving collaborative filtering schemes." 2013, pp. 1085- 1108. 